\title{
Left Atrial Thrombus Mimicking Myxoma Secondary to Rebound Hypercoagulable State
}

\author{
Muhammad Niazi ${ }^{a, d}$, Danyal Khan ${ }^{\mathrm{a}}$, Ahmad Mustafa ${ }^{\mathrm{a}}$, Abdullah B. Munir ${ }^{\mathrm{b}}$, \\ Boutros Karam ${ }^{\mathrm{b}}$, Stavros T. Snyder ${ }^{\mathrm{b}}$, James Lafferty $\mathrm{b}, \mathrm{c}$
}

\begin{abstract}
Novel oral anticoagulants (NOACs) have made the use of anticoagulation in patients with atrial fibrillation (AFib) much more convenient and predictable. It is a very common practice to hold anticoagulation either in anticipation of surgical procedures (to prevent excessive bleeding) or in case of acute bleeding episodes. This abrupt withdrawal of anticoagulation tilts the balance in favor of pro-thrombotic state in the body. We present a case of a 60 -year-old man with past medical history of AFib who abruptly discontinued his apixaban (Eliquis). This patient presented to emergency room with the complaint of weakness and numbness of left arm and left half of the face. Stroke code was activated, and patient received tissue plasminogen activator (t-PA). This resulted in significant improvement of symptoms within hours of receiving treatment. Later during the same day, patient developed numbness and tingling of fingers of left hand. On physical exam, left hand was cold to touch and radial pulse was absent. Arterial duplex revealed occluded ulnar and radial arteries. Vascular surgery performed embolectomy to establish blood flow in the radial and ulnar arteries. Transthoracic echocardiogram revealed large left atrial mass attached to the atrial septum. Most likely t-PA infusion in the setting of atrial mass led to distal showering of emboli resulting in stenosis of radial and ulnar arteries. The left atrial mass was most likely thrombus as patient had cardiac imaging including transthoracic echocardiogram and computed tomography of heart prior to these events which did not reveal any mass in left atrium. In short, as clinicians we should be aware of the hypercoagulability associated with withdrawal of anticoagulation. In addition, we should be wary of the challenges associated with differentiating cardiac masses of different etiology.
\end{abstract}

Keywords: Apixaban; Hypercoagulability; Stroke; Atrial thrombus; Myxoma; Arterial embolus

Manuscript submitted January 20, 2021, accepted February 5, 2021

Published online March 24, 2021

aDepartment of Internal Medicine, Staten Island University Hospital/Northwell Health, 475 Seaview Ave., Staten Island, NY 10305, USA

bDepartment of Cardiology, Staten Island University Hospital/Northwell Health, 475 Seaview Ave., Staten Island, NY 10305, USA

'Donald and Barbara Zucker School of Medicine at Hofstra/Northwell, Hempstead, NY, USA

${ }^{\mathrm{d} C o r r e s p o n d i n g ~ A u t h o r: ~ M u h a m m a d ~ N i a z i, ~ D e p a r t m e n t ~ o f ~ I n t e r n a l ~ M e d i c i n e, ~}$ Staten Island University Hospital/Northwell Health, 475 Seaview Ave., Staten Island, NY 10305, USA. Email: mniazi@northwell.edu

doi: https://doi.org/10.14740/jmc3656

\section{Introduction}

Novel oral anticoagulants (NOACs) have made the use of anticoagulation for thromboembolic prevention more convenient in patients with atrial fibrillation (AFib). This has made the routine use of these medications very common. As physicians, we come across this situation a number of times where we have to hold the anticoagulation when the patient is going for an invasive procedure or there is uncontrolled bleeding. One of the adverse effects of holding NOACs therapy for longer period of time is the "rebound hypercoagulation". This is something that is often overlooked in routine practice. We describe a case, where discontinuation of apixaban resulted in a large thrombus formation in left atrium which not only led to ischemic stroke but also brachial artery thrombosis requiring embolectomy. It was a challenging to differentiate large left atrial thrombus from myxoma, as the thrombus was not in left atrial appendage rather it was close to septal wall.

\section{Case Report}

A 60-year-old man with the past medical history of AFib, pulmonary embolism (PE) and hypertension presented to the emergency room (ER) with the presenting complaint of weakness and numbness of left arm and left half of the face that started approximately $90 \mathrm{~min}$ prior to the presentation. The patient was taking apixaban (5 mg twice daily) for his AFib. However, mistakenly patient stopped taking apixaban after he was told by his primary medical doctor to stop losartan. On arrival to ER, stroke code was called given patient's symptoms and his National Institutes of Health stroke scale (NIHSS) score in ER was 3. Being in the therapeutic window, the patient received intravenous tissue plasminogen activator (t-PA) bolus in ER which led to significant improvement in the symptoms. Later that day, the patient developed new onset numbness and tingling of his left hand with cold fingers as compared to the right hand. On assessment, patient was found to have a good brachial pulse, but the radial pulse was not palpable on the left side. An arterial duplex of the left arm was ordered, which revealed diminished blood flow in the brachial artery and occluded ulnar and radial arteries. After getting approval from neurology, vascular surgery performed brachial artery embolectomy, which was $24 \mathrm{~h}$ after he received t-PA. Patient was started on heparin infusion and blood flow to the distal 


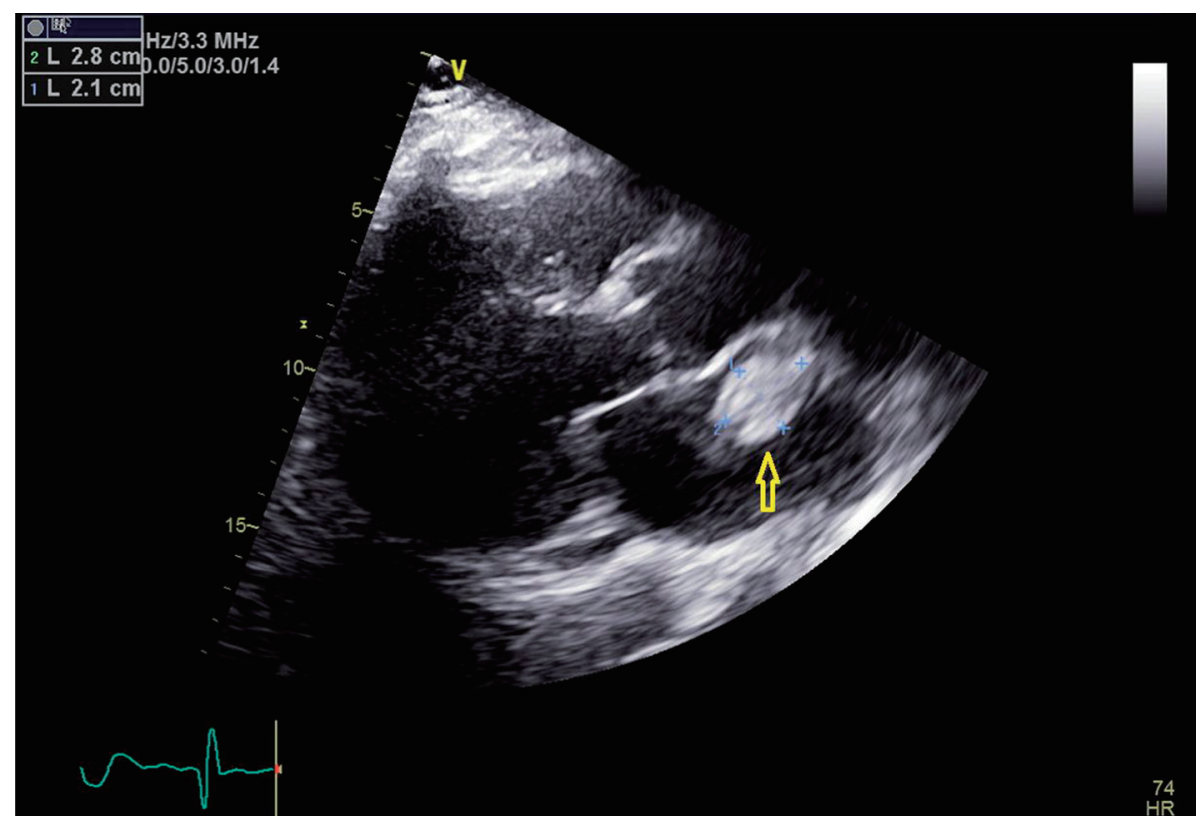

Figure 1. Transthoracic echocardiogram image with parasternal long axis view. Yellow arrow points towards the left atrial mass.

extremity was adequately established. For the further workup of stroke, a transthoracic echocardiogram was done to rule out cardio-embolic source for the acute stroke and radial arterial occlusion. Surprisingly, the patient was found to have a $2.1 \times$ $2.8 \mathrm{~cm}$ large mobile mass in the left atrium that was attached to the atrial septum (Figs. 1-3).

This was a new finding as patient had transthoracic echocardiogram and computed tomography 2 months prior to this event and there was no evidence of left atrial mass at that time. Given the acute nature of development of this mass, it was presumed to be a thrombus. Repeat echocardiogram was performed on day 6 of hospital stay and showed the similar left atrial thrombus attached with the atrial septum, unchanged from the prior study. Patient was bridged to coumadin with heparin infusion during hospital stay and discharged home safely on coumadin after a total hospital stay of 1 week.

\section{Follow-up and outcomes}

After getting discharged from hospital, patient was followed up and workup for thrombophilia was done that came back

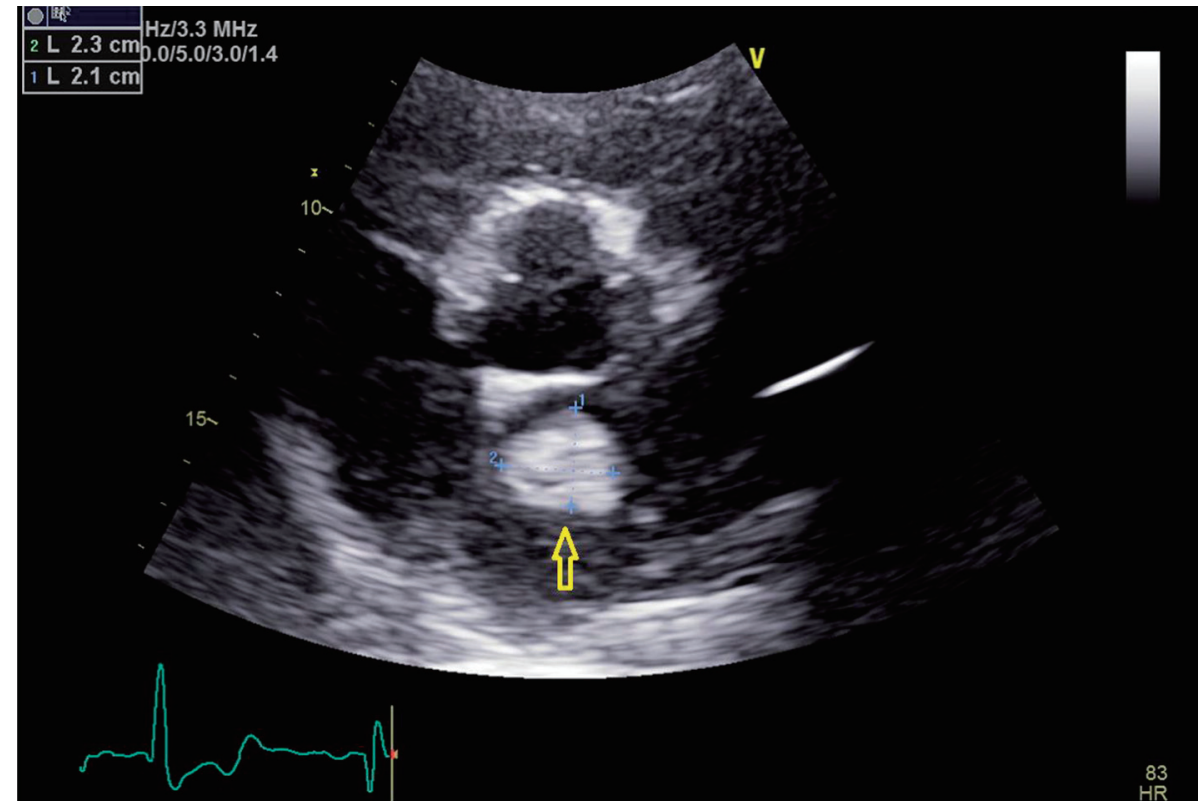

Figure 2. Transthoracic echocardiogram image with parasternal short axis view. Yellow arrow points towards the left atrial mass. 


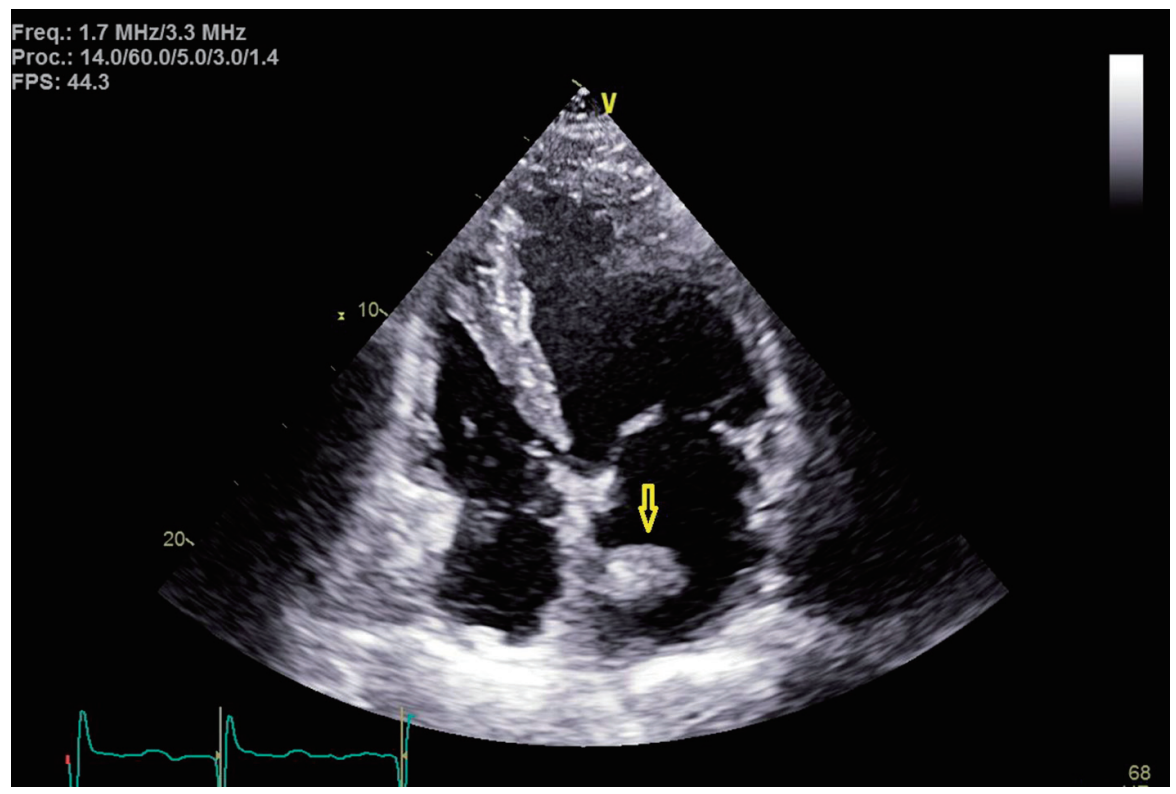

Figure 3. Transthoracic echocardiogram image with apical four-chamber view. Yellow arrow points towards the left atrial mass at the septal wall.

negative. Patient had normal protein $\mathrm{C}$ and $\mathrm{S}$ activity, normal anticardiolipin IgG and IgM antibody level and negative beta-2 glycoprotein antibody assay. Patient was negative for factor $\mathrm{V}$ Leiden mutation and prothrombin gene mutation. Patient was advised to get a repeat echocardiogram. Unfortunately, patient lost follow-up because of pandemic crisis. An echocardiogram was repeated after 1 year that showed complete resolution of the thrombus in the left atrium (Fig. 4).

\section{Discussion}

Apixaban is one of the novel oral anticoagulants agents that have been approved for the use of prophylaxis of stroke in patients with non-valvular AFib. Its mechanism of action involves the inhibition of both free factor $\mathrm{X}$-a and factor $\mathrm{X}-\mathrm{a}$ in complex with its cofactor $\mathrm{V}$-a in prothrombinase complex. It is found to be much more convenient as compared to warfarin since it does not need monitoring to ensure its therapeutic levels. Whereas response is quicker and much more predictable, there is an increased risk of thrombosis upon abrupt discontinuation of apixaban when compared to warfarin [1].

Late in the 2011, rivaroxaban was one of the first oral nonvitamin $\mathrm{K}$ antagonists that were approved to be used for nonvalvular AFib after proven non-inferior to warfarin in stroke prophylaxis among non-valvular AFib patients. This was based largely on the results of phase III trials of ROCKET-AF trials (Rivaroxaban, One-Daily, Oral, Direct Factor Xa inhibi-

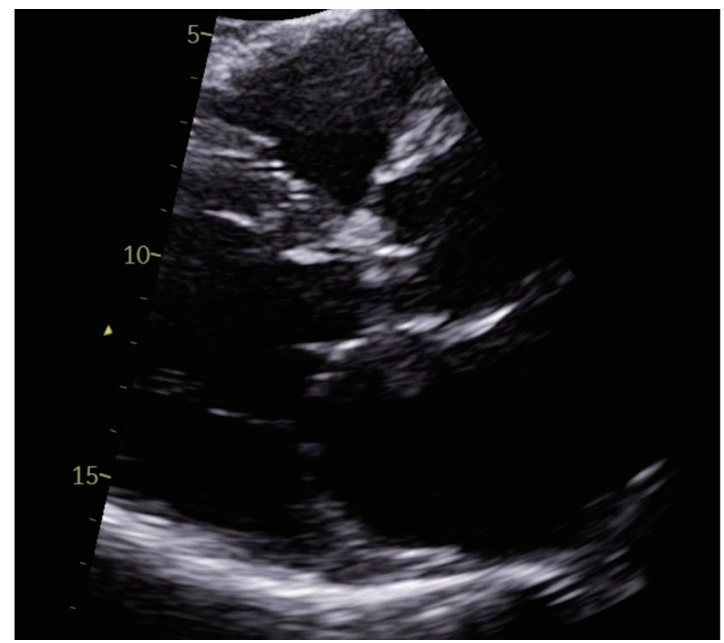

Figure 4. Repeat transthoracic echocardiogram with apical four-chamber view (left) and parasternal short axis view (right), illustrating complete resolution of left atrial thrombus. 
tion Compared with Vitamin K inhibition for Prevention of Stroke and Embolism Trial in Atrial Fibrillation) [2]. In contrast, post-hoc analysis of same trial revealed an increased risk of thrombosis seen in the patients on rivaroxaban as compared to warfarin after the discontinuation of medication in their first 3 - 30 days, when they were transitioned back to openlabel vitamin $\mathrm{K}$ antagonist (VKA) therapy. There is no clear consensus on the etiology for this transient hypercoagulability after the discontinuation of oral anticoagulant. There is a temporary increase in total fibrin and thrombin formation in these patients after the medication is discontinued which creates a pro-thrombotic state [3]. In case of stroke, these patients are shown to present with poor prognosis and a higher NIHSS score $[4,5]$. Similarly post-hoc analysis of ROCKET-AF trial showed an increased risk of cerebrovascular events after discontinuation of oral rivaroxaban in ensuing 30 days [6]. This risk persists when these oral anticoagulants are stopped transiently for an invasive procedure or due to bleeding risk. In fact, there is an increased thromboembolic risk when anticoagulation is stopped for increased bleeding risk [7, 8]. Most of the studies on factor Xa inhibitors regarding "rebound hypercoagulability" after their abrupt discontinuation have been done on rivaroxaban mainly [9]. Whereas effects of apixaban discontinuation were studied mainly in the ARISTOTLE trial when the study population was transitioned to VKA. Here an increased risk of bleeding and thromboembolism was observed but it was mainly attributed to initiation of VKA rather than apixaban [1]. However, in light of above-mentioned findings, Food and Drug Administration has a black box warning sign for increased risk of thrombosis after discontinuation of apixaban.

Getting back to our patient, he was on apixaban that was abruptly discontinued. The cardiac mass looked like a classic cardiac myxoma (Figs. 1-3) in the setting of anatomy and recent stroke, but absence of any constitutional symptoms and negative imaging 2 months prior to this presentation makes it unlikely for the mass to be myxoma. Since it is very unusual for a cardiac tumor to grow that fast in a period of months, this atrial mass was attributed as a thrombus. Furthermore, lack of sign of infection with negative blood cultures and its location on low-flow atrial walls makes cardiac vegetation less likely. Diagnosis of left atrial mass is challenging. Computed tomography [10], magnetic resonance imaging [11] and contrast echocardiography [12] can be used to visualize the mass better for diagnostic purposes but they all have limitations and can misdiagnose left atrial thrombus for myxoma. Biopsy of the mass with histopathological assessment remains the gold standard. Literature review by Mahmoud et al showed 24 cases of left atrial thrombi misdiagnosed as myxoma [13]. Of these cases, 20 patients had underlying hypercoagulable state [13]. In our patient, administration of t-PA for the stroke most likely led to showering of emboli from the atrial thrombus leading to brachial artery thrombosis from cardiac thrombo-embolism. The management of left atrial thrombus usually depends upon the characteristics of the thrombus. Although there are studies about management of left atrial thrombus with thrombolysis followed by anticoagulation (with heparin infusion) $24 \mathrm{~h}$ after t-PA infusion. But the decision mainly depends upon the characteristics of thrombus, as with an old, well-organized throm- bus with thin stalk, there is increased risk of fragment shedding after thrombolysis. Whereas, in case of new, smooth walled, mobile mass, the chance of fragment shedding is relatively less [14]. The decision of anticoagulation after thrombolysis also proves to be clinical conundrum due to increased risk of bleeding in post t-PA patients.

Thrombolytic therapy with t-PA is used in stroke patients who are within the therapeutic window $(3-4.5 \mathrm{~h}$ since the start of symptoms) and do not have any contraindications to it. However, there is evidence that t-PA can itself turn the balance towards coagulation by inducing secondary activation of coagulation cascade and inhibition of endogenous fibrinolysis [15]. Therefore, adjuvant anti-coagulation after $24 \mathrm{~h}$ of t-PA induction was recommended in older guidelines [15], but it was later abolished because of scarcity of evidence. There is at least one reported case of patient developing ventricular thrombus after the infusion of t-PA, which was treated with heparin infusion and later resolved on its own, as seen on echocardiography performed later [16]. But this possibility seems less likely in our case, since the thrombus was observed within $24 \mathrm{~h}$ of t-PA infusion. And the recent withdrawal of apixaban causing rebound hypercoagulability seems to be a more plausible explanation for the thrombus.

\section{Learning points}

Diagnosis of left atrial mass is challenging without histopathological analysis of biopsy specimen. Possibility of left atrial thrombus should be considered for patients with hypercoagulable state. With increased use of NOACs, we should be weary of rebound hypercoagulable state secondary to abrupt withdrawal.

\section{Acknowledgments}

We wish to record our deep sense of gratitude and deep thanks to Dr. Mitchell D. Weinberg, M.D., the Chairman Department of Cardiology, Staten Island University Hospital, NY for his keen interest, inspiring guidance, constant encouragement with our work during all the stages.

\section{Financial Disclosure}

None to declare.

\section{Conflict of Interest}

None to declare.

\section{Informed Consent}

Patient was contacted during the hospital stay and after the discharge. Consent was obtained over the telephone for the use 
of patient data and imaging studies for the publication of case for purely educational and research purposes to which the patient agreed. Written consent could not be obtained due to the patient's limited visitations to outpatient clinic for follow-up.

\section{Author Contributions}

Dr. Khan was part of the primary team of the patient during his care in the ICU. He came up with idea of writing this case and wrote the case presentation. Dr. Mustafa helped with writing the introduction and abstract of the case reports. Dr. Niazi did the literature review and completed the discussion part. Dr. Karam and Dr. Munir who are cardiology fellows helped with procurement of echocardiogram images and guided in the process of writing the case report. Dr. Snyder and Dr. Lafferty reviewed the final version of write up and made some changes to it according to their expertise.

\section{Data Availability}

Any inquiries related to supporting data availability of this study should be directed to corresponding author.

\section{References}

1. Granger CB, Lopes RD, Hanna M, Ansell J, Hylek EM, Alexander JH, Thomas L, et al. Clinical events after transitioning from apixaban versus warfarin to warfarin at the end of the Apixaban for Reduction in Stroke and Other Thromboembolic Events in Atrial Fibrillation (ARISTOTLE) trial. Am Heart J. 2015;169(1):25-30.

2. Patel MR, Mahaffey KW, Garg J, Pan G, Singer DE, Hacke W, Breithardt G, et al. Rivaroxaban versus warfarin in nonvalvular atrial fibrillation. $\mathrm{N}$ Engl J Med. 2011;365(10):883-891.

3. Genewein U, Haeberli A, Straub PW, Beer JH. Rebound after cessation of oral anticoagulant therapy: the biochemical evidence. Br J Haematol. 1996;92(2):479-485.

4. Park JH, Han SW, Lee KY, Choi HY, Cheon K, Cho HJ, Jung $\mathrm{YH}$, et al. Impact of non-vitamin $\mathrm{k}$ antagonist oral anticoagulant withdrawal on stroke outcomes. Front Neurol. 2018;9:1095.

5. Vene N, Mavri A, Gubensek M, Tratar G, Vizintin Cuderman T, Pohar Perme M, Blinc A. Risk of thromboembolic events in patients with non-valvular atrial fibrillation after dabigatran or rivaroxaban discontinuation - data from the ljubljana registry. PLoS One. 2016;11(6):e0156943.
6. Patel MR, Hellkamp AS, Lokhnygina Y, Piccini JP, Zhang Z, Mohanty S, Singer DE, et al. Outcomes of discontinuing rivaroxaban compared with warfarin in patients with nonvalvular atrial fibrillation: analysis from the ROCKET AF trial (Rivaroxaban Once-Daily, Oral, Direct Factor Xa Inhibition Compared With Vitamin K Antagonism for Prevention of Stroke and Embolism Trial in Atrial Fibrillation). J Am Coll Cardiol. 2013;61(6):651-658.

7. Qureshi W, Mittal C, Patsias I, Garikapati K, Kuchipudi A, Cheema G, Elbatta M, et al. Restarting anticoagulation and outcomes after major gastrointestinal bleeding in atrial fibrillation. Am J Cardiol. 2014;113(4):662-668.

8. Eikelboom JW, Mehta SR, Anand SS, Xie C, Fox KA, Yusuf S. Adverse impact of bleeding on prognosis in patients with acute coronary syndromes. Circulation. 2006;114(8):774-782.

9. Nagasayi S, Varman S, Ting YY, Ang W. Rivaroxaban withdrawal and rebound hypercoagulability leading to upper extremity deep vein thrombosis: a case report. Age Ageing. 2017;46(5):870-871.

10. Scheffel H, Baumueller S, Stolzmann P, Leschka S, Plass A, Alkadhi H, Schertler T. Atrial myxomas and thrombi: comparison of imaging features on CT. AJR Am J Roentgenol. 2009;192(3):639-645.

11. Fussen S, De Boeck BW, Zellweger MJ, Bremerich J, Goetschalckx K, Zuber M, Buser PT. Cardiovascular magnetic resonance imaging for diagnosis and clinical management of suspected cardiac masses and tumours. Eur Heart J. 2011;32(12):1551-1560.

12. Mansencal N, Revault-d'Allonnes L, Pelage JP, Farcot JC, Lacombe P, Dubourg O. Usefulness of contrast echocardiography for assessment of intracardiac masses. Arch Cardiovasc Dis. 2009;102(3):177-183.

13. Mahmoud O, Haynos W, Rollor J. Left atrial thrombi masquerading as myxomas: mini case series and literature review. CASE (Phila). 2020;4(4):252-259.

14. Lee CH, Chen CC, Chern MS. Thrombolytic therapy for acute left atrial thrombus formation in one patient with heart failure and atrial fibrillation. Circ J. 2007;71(4):604607.

15. Fassbender K, Dempfle CE, Mielke O, Schwartz A, Daffertshofer M, Eschenfelder C, Dollman M, et al. Changes in coagulation and fibrinolysis markers in acute ischemic stroke treated with recombinant tissue plasminogen activator. Stroke. 1999;30(10):2101-2104.

16. Doepp F, Sanad W, Schreiber SJ, Baumann G, Borges AC. Left ventricular apical thrombus after systemic thrombolysis with recombinant tissue plasminogen activator in a patient with acute ischemic stroke. Cardiovasc Ultrasound. 2005;3:14. 